\title{
High-resolution detection of recurrent aberrations in lung adenocarcinomas by array comparative genomic hybridization and expression analysis of selective genes by quantitative PCR
}

\author{
HONG ZHU ${ }^{1}$, MARIA PIK WONG ${ }^{2}$ and VICKY TIN ${ }^{2}$ \\ ${ }^{1}$ Department of Pathology, Beijing Friendship Hospital, Capital Medical University, Beijing 100050; \\ ${ }^{2}$ Department of Pathology, The University of Hong Kong, Hong Kong, SAR, P.R. China
}

Received January 26, 2014; Accepted March 28, 2014

DOI: $10.3892 /$ ijo.2014.2384

\begin{abstract}
Genomic abnormalities are the hallmark of cancers and may harbor potential candidate genes important for cancer development and progression. We performed array comparative genomic hybridization (array $\mathrm{CGH}$ ) on 36 cases of primary lung adenocarcinoma (AD) using an array containing $2621 \mathrm{BAC}$ or PAC clones spanning the genome at an average interval of $1 \mathrm{Mb}$. Array CGH identified the commonest aberrations consisting of DNA gains within 1p, 1q, 5p, 5q, 7p, 7q, $8 \mathrm{q}, 11 \mathrm{q}, 12 \mathrm{p}, 13 \mathrm{q}, 16 \mathrm{p}, 17 \mathrm{q}, 20 \mathrm{q}$, and losses with 6q, 9p, 10q and $18 \mathrm{q}$. High-level copy gains involved mainly 7p21-p15 and 20q13.3. Dual color fluorescence in situ hybridization (FISH) was performed on a selective locus for validation of array CGH results. Genomic aberrations were compared with different clinicopathological features and a trend of higher number of aberrations in tumors with aggressive phenotypes and current tobacco exposure was identified. According to array CGH data, 23 candidate genes were selected for quantitative PCR (qPCR) analysis. The concordance observed between the genomic and expression changes in most of the genes suggested that they could be candidate cancer-related genes that contributed to the development of lung AD.
\end{abstract}

\section{Introduction}

Lung cancer is the most frequent cause of cancer-related death worldwide, according for $\sim 1.4$ million deaths per year (1). Adenocarcinoma (AD) predominates over squamous, large cell or small cell carcinomas as the major tumor type and involves different carcinogenic mechanisms besides tobacco

Correspondence to: Dr Hong Zhu, Department of Pathology, Beijing Friendship Hospital, Capital Medical University, 95 Yong'an Road, Beijing 100050, P.R. China

E-mail: zzhuhong@hotmail.com

Key words: array CGH, lung adenocarcinoma, genomic aberration, gene expression, real-time qPCR genotoxicity. The identification of multiple genetic abnormalities which drive oncogenic signaling pathways, such as EGFR mutations and ALK fusions, has led to the development of new targeted therapies in a subset of patients $(2,3)$. However, the molecular mechanism in many AD patients still remains to be understood.

Array comparative genomic hybridization (array $\mathrm{CGH}$ ) facilitates gene discovery by enabling precise mapping of the clones of interest to specific locations on the human genome map. With increasing density of clone coverage of the genome, it has been shown that small DNA aberrations at sub-megabase levels can be identified (4). Our previous analyses on lung ADs with metaphase $\mathrm{CGH}$ have demonstrated several typical genomic aberrations. However, aberrations $<10-20 \mathrm{Mb}$ could be difficult to identify and little is known about the candidate tumor genes residing in them.

In the present study, we detected 36 lung AD cases by array $\mathrm{CGH}$ to identify genomic imbalances, using an array that consists of BAC or PAC clones covering the whole genome at an average of $1 \mathrm{Mb}$ interval. Then comparison between patient groups was also performed to identify aberrations associated with clinical and tumor characteristics. Furthermore, 23 selected genes according to array data were studied by real-time quantitative PCR (real-time qPCR) to identify the potential candidate genes that could play a critical role in $\mathrm{AD}$ progression.

\section{Materials and methods}

Study samples. Resection specimens of 36 ADs were recruited for study with written informed consent at the Grantham Hospital, Hong Kong after institutional review board approval. All patients were ethnic Chinese, and none had received any preoperative radiation therapy or chemotherapy. Demographic and clinical data were collected by the designated clinician. Non-smokers (NS) were patients who had smoked $<100$ cigarettes in the life-time; ex-smokers (EX) were those who had consumed $>100$ cigarettes but who had stopped smoking for at least one year before recruitment; current smokers (SM) were patients who were still smoking at the time of recruitment; passive smokers (PS) were those regularly exposed to environmental tobacco smoke at home or at work places. Tumor 
Table I. Clinicopathological data of 36 primary lung adenocarcinomas.

duplicates that spanned the entire genome at an average density of $1 \mathrm{Mb}$ per clone (Human BAC Arrays; Spectral Genomics, Inc., Houston, TX, USA). Clone information was provided by the chip manufacturer and clone position was mapped according to the National Center for Biotechnology Information MapViewer database, build 37.2. Extracted tumor and reference DNA was treated with RNase A and digested by DpnII (New England Biolabs), followed by purification using high pure PCR product purification kit (Roche). Each of test and reference DNA $(0.5 \mu \mathrm{g})$ were, respectively, labeled by BioPrime ${ }^{\circledR}$ DNA Labeling kit (Invitrogen), modified to include dATP, dGTP and dTTP (10 nmol each), dCTP $(0.3 \mathrm{nmol})$, Cy5-dCTP or Cy3-dCTP $(0.3 \mathrm{nmol})$ and $1 \mu 1$ Klenow fragment in a $50 \mu 1$ reaction volume. Unincorporated nucleotides were removed by MicroSpin G-50 columns (Amersham Pharmacia Biotech, Inc., Piscataway, NJ, USA). Probe size was optimized to 100-700 bp. Labeled test and sex-matched reference DNA were co-precipitated with human Cot-1 DNA and Salmon Sperm Testis DNA and resuspended in $50 \mu 1$ of hybridization buffer. For hybridization, the probe mix was first denatured and incubated at $37^{\circ} \mathrm{C}$ for $60 \mathrm{~min}$ to allow pre-annealing of Cot-1 DNA. It was then added to the array under a glass coverslip and hybridized for $16-18 \mathrm{~h}$ at $37^{\circ} \mathrm{C}$. Afterwards, the arrays were washed briefly in $2 \mathrm{X} \mathrm{SSC} / 0.5 \% \mathrm{SDS}, 50 \%$ formamide $/ 2 \mathrm{X}$ $\mathrm{SSC}$ at $50^{\circ} \mathrm{C}$ for $20 \mathrm{~min}, 2 \mathrm{X} \mathrm{SSC} / 0.1 \% \mathrm{NP} 40$ for $20 \mathrm{~min}$ and finally $0.2 \mathrm{X} \mathrm{SSC}$ for $10 \mathrm{~min}$ at $50^{\circ} \mathrm{C}$. The arrays were then rinsed in water and air dried. For each sample, two separate hybridizations were performed with reversed dye labeling to obtain 2 reciprocal datasets for analysis.

Hybridization signals were scanned with ScanArray 5000 (Packard BioScience) and captured images were analyzed using QuantArray 3.0 software (Packard BioChip Technologies). For each array, the Cy5/Cy3 signal intensity ratios were normalized according to the recommendations of the chip manufacturer. Briefly, the mean ratio and standard deviation (SD) of all target spots were calculated. A normalization constant was then derived from spots within the overall mean $\pm 1.5 \mathrm{SD}$ range. Readings from duplicate targets were averaged to yield a single intensity ratio. $\mathrm{Cy} 5 / \mathrm{Cy} 3$ intensity ratios of $<0.85$ or $>1.15$ were considered to show DNA copy number alterations (CNA). These threshold values were similar to those adopted in other array CGH studies of solid tumors using BAC array platforms $(5,6)$. High level CNA were designated for ratios of $<0.5$ and $>1.5$. Two repeats of normal vs. normal hybridizations with pooled normal DNA were performed to ensure consistency of criteria. For each target, the final status of DNA gain or loss was assigned only if the reciprocal ratios of the dye-reversal experiments yielded concordant results. Loci showing imbalances in the control experiments or with inconsistent map positions were omitted during data interpretation.

Fluorescence in situ hybridization (FISH). Dual color FISH analysis was performed on paraffin tumor tissue sections. PAC clone RP5-885L7 (20q13.3, $60.9 \mathrm{Mb}$ ), corresponding to loci of DNA gain by array CGH, was used as test probe. The test probes were labeled by nick translation with SpectrumOrange (Vysis, Downers Grove, IL, USA). Procedures of probe labeling and FISH analysis were according to previously published protocols (7).

Array CGH and data analysis. The DNA microarray

contained 2621 non-overlapping $\mathrm{BAC}$ and PAC clones in

\begin{tabular}{lrr} 
Gender & & \\
Female & 24 & 67 \\
Male & 12 & 33 \\
Age (years) & & \\
$<50$ & 3 & 8 \\
$50-59$ & 11 & 31 \\
$60-69$ & 12 & 33 \\
$\geq 70$ & 10 & 28 \\
Smoking history & & \\
Non-smoker & 22 & 61 \\
Current smoker & 8 & 22 \\
Ex-smoker & 2 & 6 \\
Passive smoker & 4 & 11 \\
Differentiation & & \\
Well & 18 & 50 \\
Moderate & 15 & 42 \\
Poor & 3 & 8 \\
TNM stage & & \\
I & 24 & 67 \\
II & 6 & 17 \\
III & 5 & 14 \\
IV & 1 & 3 \\
\hline
\end{tabular}

classification and grading were independently performed by 2 qualified pathologists according to the WHO classification of the 36 patients were shown in Table I. and kept at $-70^{\circ} \mathrm{C}$ until use. Haematoxylin and eosin stained sections were examined histologically prior to DNA and preserved morphology, low stromal and inflammatory cell content were dissected for study, and non-neoplastic or necrotic which DNA was extracted, pooled and used as reference DNA for array CGH. For real-time qPCR, to ensure comparability of results from different test samples, all the cDNA used in the ftudy were prepared from a fixed amount of starti after strict quality check to ensure absence of RNA degradation. All aliquots of samples and replicates of each case used in the analysis of every gene were taken from the same sample stock to ensure consistency of quality and amount of material input. 

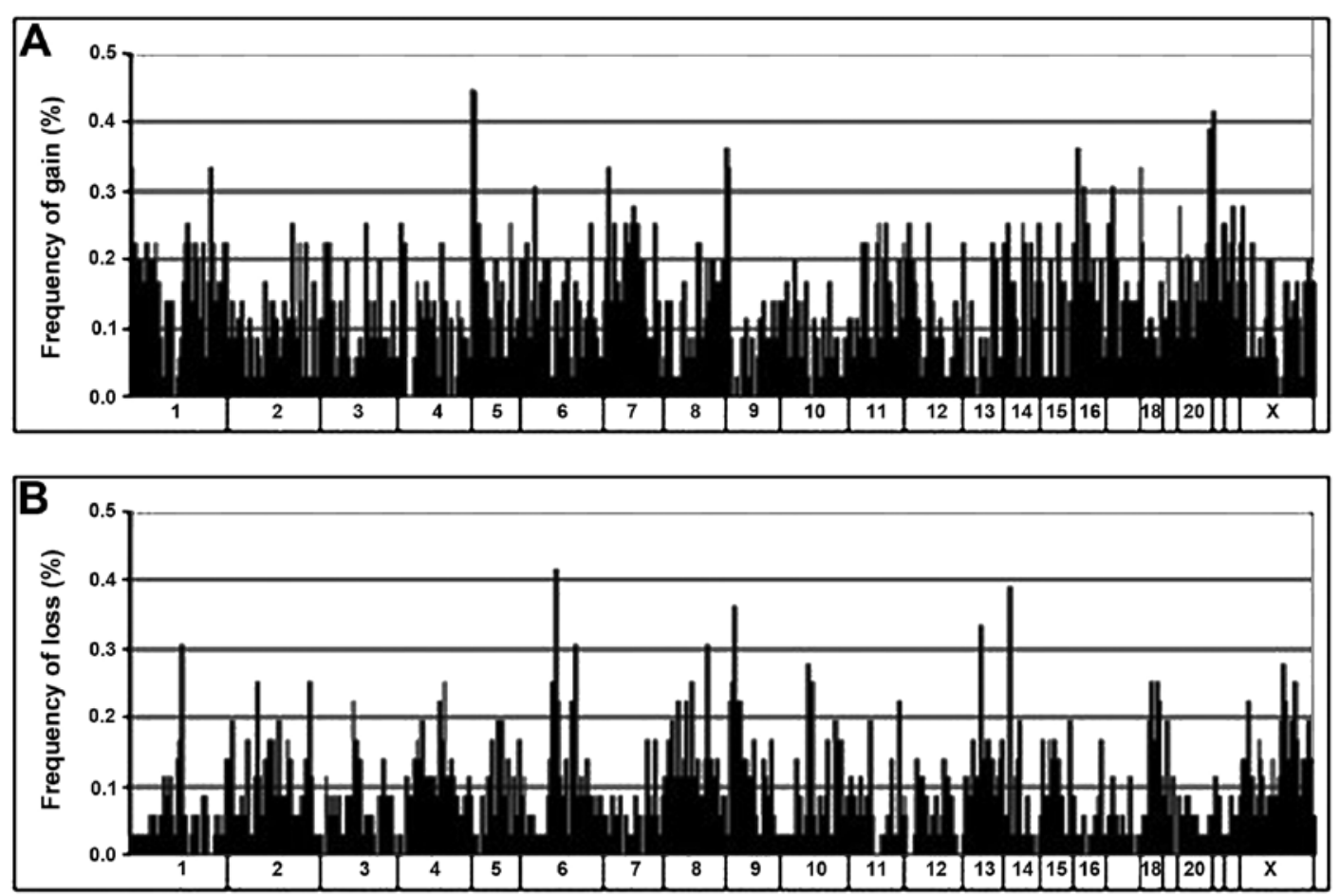

Figure 1. Copy number alteration profiles of primary lung AD by array CGH. Frequencies of DNA (A) gain and (B) loss are plotted against chromosomal positions indicated along the $\mathrm{x}$-axis.

Real-time qPCR. Twenty-three genes were selected for evaluation in the present study. These genes should be in the aberrant loci by array $\mathrm{CGH}$ and involved in biological processes with potential enhancing effects on tumor development and progression, such as cell proliferation, cell cycle control, cell death, cell adhesion and transcriptional regulation. Twenty genes were selected from regions of genomic gain and 3 genes were from frequently deleted regions.

Real-time qPCR analysis was performed using the ABI PRISM 7700 Sequence Detection system (PE Applied Biosystems). Primer and probe sets of the selected genes were commercially obtained (Assay-on demand TaqMan ${ }^{\circledR}$ Gene Expression Assays; Applied Biosystems). Samples were analyzed in duplicate and repeated for verification where appropriate. The comparative $\mathrm{Ct}$ method was used for the calculation of relative level of a test gene in a given tumor sample normalized according to $\beta 2 \mathrm{M}$ as reference for cDNA input. Results were presented as $\log _{2}$-transformed ratios between the tumor and pooled normal lung samples.

Statistical analysis. Statistical analysis was performed with the SPSS 18.0.0 software. Chi-square or Fisher's exact test was used to compare aberrant loci between tumors of different clinicopathological characteristics. Student's t-test for independent samples was used for the comparison of mean expression levels between different clinicopathological groups for individual genes. A two-sided P-value of $<0.05$ was taken as indicating a statistically significant result.

\section{Results}

Genomic CNA identified by array $C G H$. All the 36 ADs analyzed showed genomic imbalances, with a mean of
$221.6 \pm 168.4(8.5 \pm 6.4 \%)$ aberrations per tumor. Overall, DNA gains (mean, 131.6) were more frequent than losses (mean, 90.1). Graphical representation of the alteration frequencies of all loci along each chromosome is presented in Fig. 1.

The distribution of DNA imbalances was non-uniform. Clustering of aberrations was observed, where multiple altered loci were found in contiguous genomic sites or closely within the same cytogenetic band. These clustered alterations were mainly found within $1 \mathrm{p}, 1 \mathrm{q}, 5 \mathrm{p}, 5 \mathrm{q}, 7 \mathrm{p}, 7 \mathrm{q}$, $8 q, 11 q, 12 p, 13 q, 16 p, 17 q$ and $20 q$ for DNA gain and within $6 q, 9 p, 10 q$ and $18 q$ for DNA loss. Regions containing alterations in 6 or more tumors are listed in Table II. The genomic distances they spanned ranged from 0.3 to $8.4 \mathrm{Mb}$. The most frequently gained loci were within 5p15.33-p15.31 (44.4\%), 20q13.31-20q13.33 (38.9\%), 8q24.21-q24.3 (36.1\%), 16p13.3p13.12 (36.1\%), followed by $1 \mathrm{p} 36.32$, 1q32.1 and $17 \mathrm{q} 25.3$ $(33.3 \%)$. The commonest loci of loss were within 9p23 (36.1\%), followed by 6q16.3 (30.6\%).

Another pattern of CNA consisted of focal aberrations spanned by single clones which were found in almost all chromosomes. The most frequently altered loci were DNA gain involving 6p22 at 15.6 Mb (clone RP1-147M19, 30.6\%), 7p13 at $47.1 \mathrm{Mb}$ (clone $\mathrm{AC} 073341.10,27.8 \%$ ) and $22 \mathrm{q} 12$ at $31.5 \mathrm{Mb}$ (clone Z73979.1, 27.8\%); and DNA loss involving 1p21 at 103.9 Mb (clone RP11-259N12, 30.6\%), 6q12 at 67.1 Mb (clone RP11-80L16, 41.7\%), 8q21 at 86.8 Mb (RP11-96G1, 30.6\%) and $14 \mathrm{q} 12$ at $28.6 \mathrm{Mb}$ (clone RP11-125A5, 38.9\%).

High-level copy gains were identified in 16 of the 36 tumors. Nine loci showed recurrent gains involving at least 2 cases. They were focused at 2 regions, comprising 7p21.1-p15.3 (18.1 to $21.2 \mathrm{Mb}$ ) spanned by 3 clones (RP11-70K3, RP11-51L23 and CTB-23M10) and 20q13.31-q13.33 (56.6 to 62.4 Mb) spanned by 6 clones (RP5-907D15, RP5-1043L13, RP5-1107C24, 
Table II. Clustered aberrations of DNA gain (upper panel) and loss (lower panel) spanned by multiple clones detected by array $\mathrm{CGH}$ in lung adenocarcinomas.

\begin{tabular}{|c|c|c|c|c|}
\hline Cytoband & Region size & Clone name & Chromosome start position (Mb) & $\%$ Gain $(n=36)$ \\
\hline \multirow[t]{2}{*}{$1 \mathrm{p} 36.32$} & $1.4 \mathrm{Mb}$ & RP1-163G9 & chr1_3.0 & 33.3 \\
\hline & & RP11-447M5 & chr1_4.2 & 27.8 \\
\hline \multirow[t]{3}{*}{$1 \mathrm{p} 36.23-\mathrm{p} 36.21$} & $3.3 \mathrm{Mb}$ & RP11-476D13 & chr1_9.2 & 22.2 \\
\hline & & AL031984.13 & chr1_10.4 & 22.2 \\
\hline & & RP5-888M10 & chr1_12.4 & 16.7 \\
\hline \multirow[t]{3}{*}{$1 \mathrm{q} 21.2-\mathrm{q} 21.3$} & $3.3 \mathrm{Mb}$ & RP4-790G17 & chr1_147.0 & 22.2 \\
\hline & & RP11-71L20 & chr1_148.1 & 25.0 \\
\hline & & RP1-148L21 & chr1_150.3 & 22.2 \\
\hline \multirow[t]{2}{*}{$1 \mathrm{q} 25.3$} & $1.5 \mathrm{Mb}$ & RP11-63O2 & chr1_180.7 & 22.2 \\
\hline & & RP11-79I7 & chr1_182.0 & 19.4 \\
\hline \multirow[t]{5}{*}{ 1q32.1 } & $3.9 \mathrm{Mb}$ & RP11-150L7 & chr1_197.9 & 16.7 \\
\hline & & RP11-246J15 & chr1_198.9 & 16.7 \\
\hline & & RP11-335O13 & chr1_199.8 & 25.0 \\
\hline & & RP11-80N9 & chr1_201.4 & 16.7 \\
\hline & & RP11-243M13 & chr1_201.6 & 33.3 \\
\hline \multirow[t]{3}{*}{$1 q 44$} & $3.4 \mathrm{Mb}$ & RP1-241M7 & chr1_240.7 & 19.4 \\
\hline & & RP11-91C5 & chr1_242.5 & 22.2 \\
\hline & & RP11-656O22 & chr1_244.1 & 22.2 \\
\hline \multirow[t]{3}{*}{$5 \mathrm{p} 15.33-\mathrm{p} 15.31$} & $3.4 \mathrm{Mb}$ & RP11-20B3 & chr5_3.1 & 44.4 \\
\hline & & RP11-89N22 & chr5_4.1 & 36.1 \\
\hline & & AC010635.6 & chr5_6.3 & 22.2 \\
\hline \multirow[t]{5}{*}{ 5p15.31-p15.2 } & $5.0 \mathrm{Mb}$ & RP11-91M12 & chr5_9.9 & 19.4 \\
\hline & & RP11-145B1 & chr5_10.3 & 25.0 \\
\hline & & RP11-72C10 & chr5_10.7 & 19.4 \\
\hline & & RP11-79G1 & chr5_11.1 & 19.4 \\
\hline & & RP11-81P9 & chr5_14.7 & 19.4 \\
\hline \multirow[t]{3}{*}{$5 q 31.3$} & $0.8 \mathrm{Mb}$ & RP11-79K4 & chr5_140.3 & 25.0 \\
\hline & & RP11-15J20 & chr5_140.9 & 16.7 \\
\hline & & RP11-55M16 & chr5_141 & 19.4 \\
\hline \multirow[t]{4}{*}{$7 \mathrm{p} 21.1$} & $3.5 \mathrm{Mb}$ & RP11-89B15 & chr7_15.4 & 25.0 \\
\hline & & RP11-123E5 & chr7_17.3 & 19.4 \\
\hline & & RP11-70K3 & chr7_18.1 & 16.7 \\
\hline & & RP11-384L2 & chr7_18.7 & 19.4 \\
\hline \multirow[t]{3}{*}{ 7p15.1-p14.3 } & $3.5 \mathrm{Mb}$ & RP11-242I4 & chr7_30.2 & 25.0 \\
\hline & & AC018648.5 & chr7_32.6 & 22.2 \\
\hline & & RP11-89N17 & chr7_33.5 & 22.2 \\
\hline \multirow[t]{2}{*}{$7 q 11.21-q 11.22$} & $2.5 \mathrm{Mb}$ & RP11-91C6 & chr7_65.0 & 16.7 \\
\hline & & RP11-89D15 & chr7_67.4 & 25.0 \\
\hline \multirow[t]{3}{*}{$8 q 24.21-q 24.3$} & $4.2 \mathrm{Mb}$ & RP11-13A18 & chr8_141.6 & 36.1 \\
\hline & & RP11-349C2 & chr8_145.6 & 25.0 \\
\hline & & CTC-261I1 & chr8_145.7 & 33.3 \\
\hline \multirow[t]{3}{*}{$11 q 13.3-q 13.5$} & $5.0 \mathrm{Mb}$ & AP001271.4 & chr11_70.0 & 22.2 \\
\hline & & RP11-168B13 & chr11_74.8 & 22.2 \\
\hline & & RP11-91P18 & chr11_74.9 & 25.0 \\
\hline \multirow[t]{2}{*}{$11 q 14.2$} & $1.2 \mathrm{Mb}$ & RP11-80F20 & chr11_86.5 & 19.4 \\
\hline & & RP11-876F8 & chr11_87.7 & 25.0 \\
\hline \multirow[t]{3}{*}{$12 \mathrm{p} 13.33-\mathrm{p} 13.31$} & $5.3 \mathrm{Mb}$ & RP11-598F7 & chr12_0.0 & 19.4 \\
\hline & & RP11-543P15 & chr12_3.1 & 25.0 \\
\hline & & RP11-319E16 & chr12_5.2 & 19.4 \\
\hline
\end{tabular}


Table II. Continued.

\begin{tabular}{|c|c|c|c|c|}
\hline Cytoband & Region size & Clone name & Chromosome start position (Mb) & $\%$ Gain $(\mathrm{n}=36)$ \\
\hline \multirow[t]{2}{*}{ 13q31.1-q31.2 } & $0.7 \mathrm{Mb}$ & RP11-753M10 & chr13_86.4 & 19.4 \\
\hline & & RP11-29P20 & chr13_87.0 & 22.2 \\
\hline \multirow[t]{2}{*}{ 13q34 } & $3.2 \mathrm{Mb}$ & RP11-474D23 & chr13_110.8 & 22.2 \\
\hline & & RP11-245B11 & chr13_113.8 & 19.4 \\
\hline \multirow[t]{7}{*}{$16 \mathrm{p} 13.3$} & $5.9 \mathrm{Mb}$ & RP11-344L6 & chr16_0.0 & 22.2 \\
\hline & & CTB-191K2 & chr16_0.1 & 30.6 \\
\hline & & RP11-334D3 & chr16_0.9 & 36.1 \\
\hline & & RP11-417B20 & chr16_1.4 & 25.0 \\
\hline & & RP11-433P17 & chr16_3.3 & 16.7 \\
\hline & & RP11-89M4 & chr16_4.5 & 16.7 \\
\hline & & RP11-349I11 & chr16_5.7 & 16.7 \\
\hline \multirow[t]{4}{*}{ 16p13.13-16p13.12 } & $3.4 \mathrm{Mb}$ & RP11-396B14 & chr16_11.1 & 19.4 \\
\hline & & RP11-81F1 & chr16_11.2 & 30.6 \\
\hline & & RP11-165M1 & chr16_12.3 & 19.4 \\
\hline & & RP11-91M7 & chr16_14.4 & 16.7 \\
\hline \multirow[t]{3}{*}{$17 q 25.3$} & $3.1 \mathrm{Mb}$ & RP11-165J13 & chr17_74.7 & 33.3 \\
\hline & & RP11-46E14 & chr17_75.3 & 22.2 \\
\hline & & CTB-50C4 & chr17_77.8 & 16.7 \\
\hline \multirow[t]{11}{*}{ 20q13.31-20q13.33 } & $8.4 \mathrm{Mb}$ & RP5-885A10 & chr20_54.0 & 22.2 \\
\hline & & RP4-749H19 & chr20_54.9 & 36.1 \\
\hline & & RP5-907D15 & chr20_56.6 & 25.0 \\
\hline & & RP5-1043L13 & chr20_58.2 & 38.9 \\
\hline & & RP5-1040G13 & chr20_59.1 & 33.3 \\
\hline & & RP5-1107C24 & chr20_59.9 & 30.6 \\
\hline & & RP5-1005F21 & chr20_60.1 & 30.6 \\
\hline & & RP5-885L7 & chr20_60.9 & 25.0 \\
\hline & & RP4-583P15 & chr20_61.8 & 33.3 \\
\hline & & AL118506.27 & chr20_62.0 & 25.0 \\
\hline & & AL137028.9 & chr20_62.4 & 22.2 \\
\hline Cytoband & Region size & Clone name & Chromosome start position (Mb) & $\%$ Loss $(n=36)$ \\
\hline \multirow[t]{2}{*}{$6 q 16.3$} & $0.5 \mathrm{Mb}$ & RP11-90O11 & chr6_101.9 & 30.6 \\
\hline & & RP11-79O12 & chr6_102.2 & 16.7 \\
\hline \multirow[t]{3}{*}{$9 \mathrm{p} 24.2$} & $1.6 \mathrm{Mb}$ & RP11-79M14 & chr9_2.6 & 22.2 \\
\hline & & RP11-32F11 & chr9_3.1 & 25.0 \\
\hline & & RP11-31M2 & chr9_4.1 & 16.7 \\
\hline \multirow[t]{2}{*}{$9 p 24.1$} & $1.1 \mathrm{Mb}$ & RP11-79K3 & chr9_7.3 & 16.7 \\
\hline & & RP11-376O21 & chr9_8.2 & 25.0 \\
\hline \multirow[t]{3}{*}{9 p23 } & $3.1 \mathrm{Mb}$ & RP11-91E3 & chr9_9.6 & 30.6 \\
\hline & & RP11-32D4 & chr9_11.5 & 36.1 \\
\hline & & RP11-328C23 & chr9_12.5 & 19.4 \\
\hline \multirow[t]{2}{*}{ 10q21.1 } & $4.4 \mathrm{Mb}$ & RP11-75M12 & chr10_52.8 & 27.8 \\
\hline & & RP11-79A2 & chr10_57.1 & 25.0 \\
\hline \multirow[t]{2}{*}{ 18q22.1-q22.2 } & $0.3 \mathrm{Mb}$ & RP11-90A7 & chr18_64.7 & 25.0 \\
\hline & & RP11-49H23 & chr18_64.9 & 22.2 \\
\hline
\end{tabular}

RP5-1005F21, RP5-885L7 and RP1-81F12). No locus of high level loss was detected in the tumors studied.

CNA and clinicopathological correlations. Comparison between tumors with different clinicopathological features showed a trend of higher total and mean number of genetic aberrations in tumors showing larger size ( $\geq 2 \mathrm{~cm}$ ), lymph node metastasis, higher pathological stages (stage II or higher), moderate/poor differentiation and previous or current tobacco exposure. The difference, however, was not statistically 


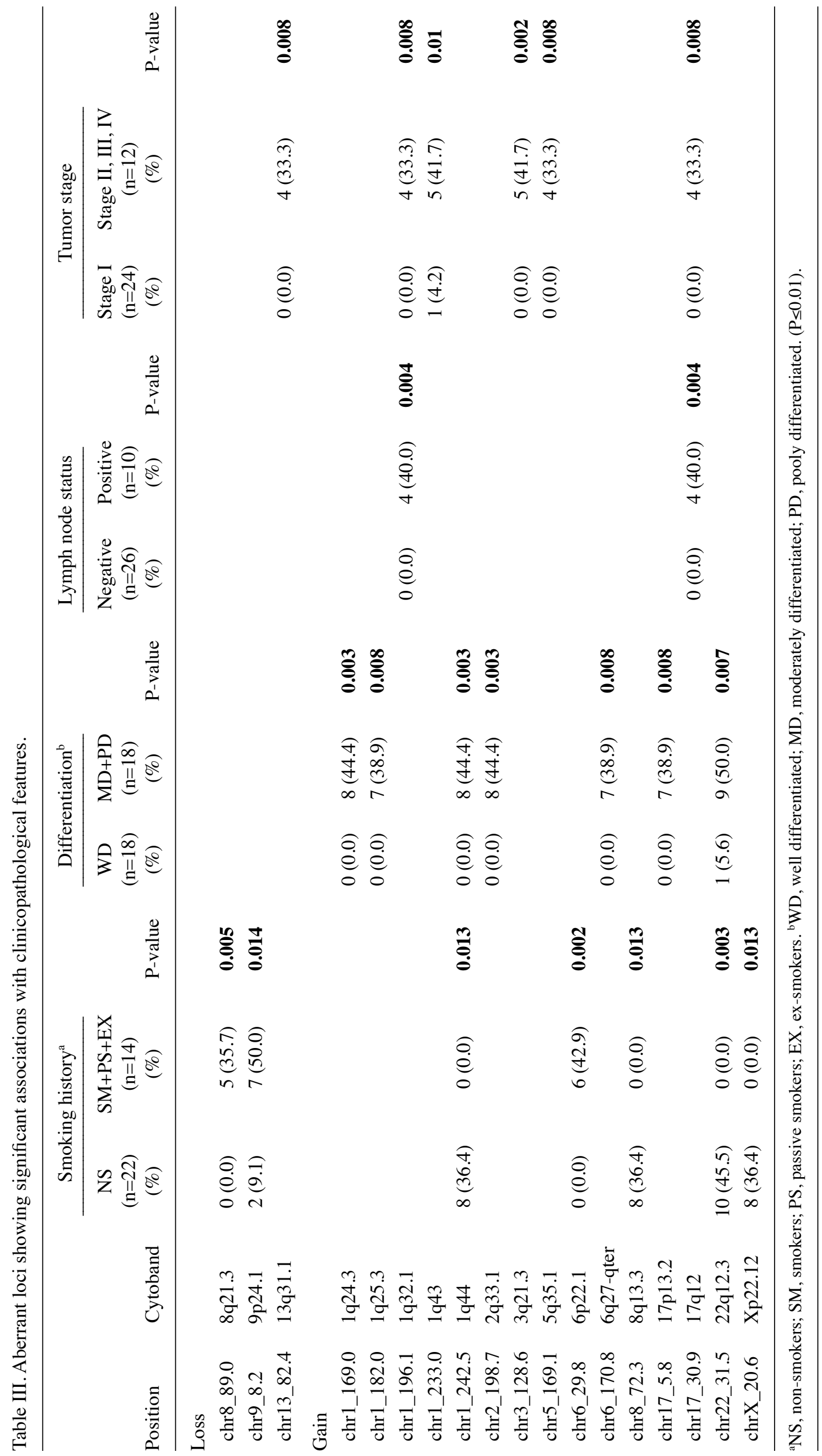




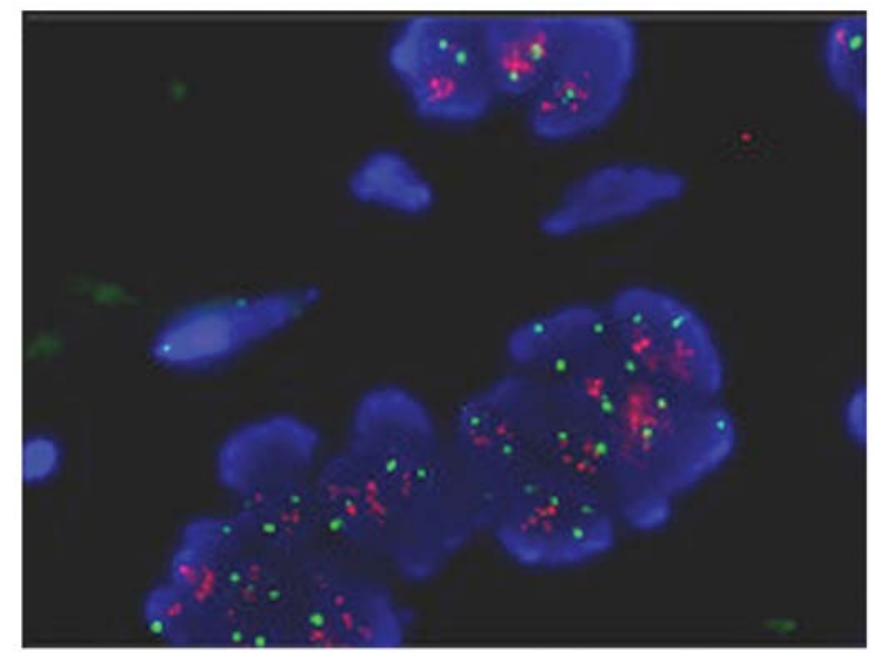

Figure 2. Dual-color FISH analysis. Increased test (RP5-885L7, 20q13.3, labeled in orange) to control (CEP-20, labeled in green) signal ratios in tumor sample.

Table IV. Candidate gene expression levels by real-time qPCR in 36 cases of lung adenocarcinomas.

\begin{tabular}{lllcc}
\hline $\begin{array}{l}\text { Gene } \\
\text { symbol }\end{array}$ & Cytoband & $\begin{array}{c}\text { Array } \\
\text { CGH }\end{array}$ & $\begin{array}{c}\text { Expression } \\
\text { level }\left(\log _{2}\right)\end{array}$ & $\begin{array}{c}\text { Validation } \\
\text { (DNA/RNA) }\end{array}$ \\
\hline CMM & $1 \mathrm{p} 36.32$ & Gain & 0.48 & Yes \\
PAX7 & $1 \mathrm{p} 36.2$ & Gain & 3.44 & Yes \\
ILF2 & $1 \mathrm{q} 21.3$ & Gain & 3.02 & Yes \\
MUC1 & $1 \mathrm{q} 22$ & Gain & 2.20 & Yes \\
ELF3 & $1 \mathrm{q} 32.1$ & Gain & 1.96 & Yes \\
CHI3L1 & $1 \mathrm{q} 32.1$ & Gain & 2.23 & Yes \\
SMYD3 & $1 \mathrm{q} 44$ & Gain & 2.99 & Yes \\
TERT & $5 \mathrm{p} 13.33$ & Gain & 4.89 & Yes \\
PRDM13 & $6 \mathrm{q} 16.3$ & Loss & $\mathbf{1 . 1 4}$ & No \\
AGR2 & $7 \mathrm{p} 21.1$ & Gain & 4.34 & Yes \\
SBDS & $7 \mathrm{q} 11.21$ & Gain & 2.35 & Yes \\
RECQL4 & $8 \mathrm{q} 24.3$ & Gain & 3.31 & Yes \\
OVC & $9 \mathrm{p} 24.2$ & Loss & $\mathbf{2 . 9 1}$ & No \\
DKK1 & $10 \mathrm{q} 21.1$ & Loss & $\mathbf{- 0 . 2 3}$ & Yes \\
CCND1 & $11 \mathrm{q} 13.3$ & Gain & 3.02 & Yes \\
ETV6 & $12 \mathrm{p} 13.33$ & Gain & 1.39 & Yes \\
GAS6 & $13 \mathrm{q} 34$ & Gain & 0.22 & Yes \\
AXIN & $16 \mathrm{p} 13.3$ & Gain & 1.79 & Yes \\
EMP2 & $16 \mathrm{p} 13.13$ & Gain & 3.56 & Yes \\
ASPL & $17 \mathrm{q} 25.3$ & Gain & 2.02 & Yes \\
STK6 & $20 \mathrm{q} 13.31$ & Gain & 2.97 & Yes \\
TFAP2C & $20 \mathrm{q} 13.31$ & Gain & 1.32 & Yes \\
EEF1A2 & 20q13.33 & Gain & 4.52 & Yes \\
\hline
\end{tabular}

significant. Tumors of more aggressive phenotypes showed more frequent alterations in $13 q$ for DNA loss, and $1 \mathrm{q}, 2 \mathrm{q}$, $3 q, 5 q$ and $17 q$ for DNA gain. Furthermore, tobacco-exposed patients showed more often loss at 8q21.3, 9p24.1 and gain at $6 \mathrm{p} 22.1$, while non-smokers showed more frequent gains at 1q44, 8q13.3, 22q12.3 and Xp22.12 (Table III).

FISH analysis. Dual-color FISH analysis of a selective loci at 20q13.3 which show high-level gain were performed in 5 of 8 amplified cases of paraffin sections. The average ratio of test to reference signal was 2.31 , which showed agreement with the results detected by array $\mathrm{CGH}$ (Fig. 2).

Real-time qPCR. For the 20 genes located in regions of DNA gain, the tumor to normal expression ratios investigated by real-time qPCR ranged from 0.22 to 4.89 . While for the 3 genes located in regions of genomic loss, the tumor to normal expression ratios were $-0.23,1.14$ and 2.91 , respectively (Table IV). The concordance observed between the genomic and expression changes for most of the genes suggested that they could be candidate oncogenes that contributed to the development of lung AD, while none of the genes showed expression levels that were significantly associated with the cliniopathological parameters.

\section{Discussion}

The present study utilized an array platform that consists of large insert DNA template clones covering the genome at an average resolution of $1 \mathrm{Mb}$ to characterize the profiles of genomic imbalances in primary lung AD. Recurrent aberrations were found clustered in regional distribution spanned by multiple clones, and in focal distribution spanned by single clones. These loci correlated with results of our previous array $\mathrm{CGH}$ study performed on non-small cell lung cancer (NSCLC) cell lines, and corresponded to well-known aberrations of lung cancers defined by other array CGH studies $(8,9)$. The common patterns of genomic changes indicate that lung cancer-related genes are likely to be contained within these hot spots of alterations.

$5 \mathrm{p} 15.33$ is the most frequently gained locus in the present study. A pooled analysis from East Asia totaling 1164 lung ADs revealed that genetic variation in the CLPTM1LTERT locus of 5p15.33 was directly associated with the risk of lung cancer, most notably AD (10). Other studies also found 5 p15.33 locus influence lung cancer risk $(11,12)$. The second frequently gained region involves an $8.4-\mathrm{Mb}$ segment of gain at 20q13.31-q13.33 (56.5 to 62.1 Mb). This region is noteworthy as it is one of the commonest aberrations in lung cancer as well as tumors of the gastric cancer and pancreatic cancer (13-15). The FISH analysis on tumor samples confirmed modest DNA gains. The third altered locus of 8q24.21-q24.3 is a known site of DNA gains and amplifications and contains the c-Myc oncogene. Different array $\mathrm{CGH}$ studies have found aberrant loci correlating to this region $(16,17)$. The detection of these multiple loci by independent studies suggests the presence of multiple oncogenes at these regions.

Comparison of tumors of different clinicopathological groups was performed to identify genomic markers of potential diagnostic and prognostic importance. All the aberrant loci that showed statistical significance were more common in tumors of more aggressive phenotypes, i.e., poor differentiation, lymph node metastasis and higher disease stage. This 
observation corroborates the concept that tumor progression is enhanced by genomic instability and accumulation of genetic abnormalities.

Several studies have evaluated DNA alterations in tumors of different tobacco exposure using different approaches. However, the chromosomal regions characterizing tobaccoor non tobacco-induced lung AD remain unclear. By cluster analysis of genomic aberrations in $55 \mathrm{AD}$ detected by a cancer gene-rich array, Shibata et al (18) identified subgroups associated with female non-smokers, male current or previous smokers and a group with mixed gender and tobacco history. Loci of DNA gains on 1p, 4p, 11p, 12q, 16p, 17q, 19q, 20p, $20 q$ and $22 q$, and losses on $1 p, 6 q, 10 q, 13 q, 15 q$ and $18 p$ were associated with non-smokers, while loci of $19 q$ gain and $22 q$ loss were associated with smokers. Our results cannot be directly compared since our array did not specifically span tumor genes. Nevertheless, 22q12.3 (31.5 Mb) which shows DNA gain in 10 of 22 non-smokers compared to 0 of 14 tobacco-exposed tumors $(\mathrm{P}=0.003)$ is located close to one of their reported loci (22q12.2 gain) in non-smokers. Analysis of a larger number of tumors and the use of identical, highresolution array platforms in different populations would be necessary for definitive identification of genomic markers associated with different tobacco exposure and clinicopathological profiles.

It is a common assumption that chromosomal gains and losses have 'dosage effects' on the expression of at least some of the genes within these regions, which should be good candidates for involvement in carcinogenesis. The concordance observed between the genomic changes and expression level by real-time qPCR for the most selected genes in the present study suggested that they could be candidate oncogenes or tumor suppressor genes that contributed to the development of lung AD. Some of them had been investigated in clinical or in vitro studies and results supported their putative oncogenic roles. For example, PAX7 gene on 1pter-p33 that encodes a transcription factor was most amplified in lung squamous cell carcinoma (19), and elevated expression of MUC1 on 1q22 contributed to smoking-induced lung cancers that were driven by inflammatory signals of macrophages (20). One of Stat3 downstream gene products, chitinase 3-like 1 (CHI3L1, in region 1q32.1) protein, was found to be a potential biomarker of inflammation-induced lung cancer by a study of lung tumor mouse models (21). TERT was locused on 5p15.33 which was one of the susceptibility loci from a genome-wide association study of lung cancer in never-smoking Asian women (8), and was the highest expressed gene in the present study. Several studies using animal models and human NSCLC tissues have reported that TERT mRNA and protein were overexpressed in lung tumors compared with normal lung tissues $(22,23)$. The 7p genes AGR2 was revealed to be significantly overexpressed in lung AD by comparison with SCCs or normal lung tissue (24). Among the 3 genes located in the loss region, only Dickkopf1 (DKK1, in region 10q21.1) was underexpressed compared to normal lung tissue. DKK1 is the predominant secretory antagonist of the Wnt/ $\beta$-catenin signal, suggesting that if DKK1 were not precisely regulated, it could result in tumor formation and progression. In fact, several clinical studies demonstrated that DKK1 was downregulated in melanoma and breast cancer $(25,26)$. The observation that
CCND1 (in region 11q13.3) was amplified and overexpressed in a fraction of NSCLC when compared with normal lung was strong evidence implicating the inappropriate expression of CCND1 in lung carcinogenesis (27). Amongst genes located in the recurrently gained $20 \mathrm{q} 13.3$ region, TFAP2C not only were highly expressed in breast cancer, but also associated with reduced survival (28). And EEF1A2 were amplified or overexpressed in various cancers including NSCLC which highlights its oncogenic potential (29).

None of the genes showed expression levels that were significantly associated with the clinicopathological parameters, indicating that the currently studied genes were not likely candidates that mediated the differences in tumor differentiation and behavior implicated by the array CGH analysis. The possible explanations for failing to demonstrate these relations could be that gene-mining based on the strategy of genomic, expressional and functional correlations was insufficient in the clinical context, reflecting the complexity of genetic and transcriptional interactions in tumor development. Moreover, since lung ADs are known to consist of heterogeneous tumor populations, it might be worthwhile to conduct the investigation in a larger sample of well controlled, homogeneous tumor population when a candidate tumor gene with strong evidence is considered.

In conclusion, we used array $\mathrm{CGH}$ to identify loci of frequent DNA aberrations in primary lung AD. Real-time qPCR confirmed cancer-related genes in regions with CNA from array $\mathrm{CGH}$ data. The limitations of the present study are the small number of samples and further studies are needed to validate our results. The identified aberrations and candidate genes can be used as starting points for more specific investigations for pathogenesis in primary lung $\mathrm{AD}$, as well as in tumors of different etiological or phenotypic characteristics.

\section{References}

1. Jemal A, Bray F, Center MM, Ferlay J, Ward E and Forman D: Global cancer statistics. CA Cancer J Clin 61: 69-90, 2011.

2. Paez JG, Jänne PA, Lee JC, et al: EGFR mutations in lung cancer: correlation with clinical response to gefitinib therapy. Science 304: 1497-1500, 2004.

3. Kwak EL, Bang YJ, Camidge DR, et al: Anaplastic lymphoma kinase inhibition in non-small-cell lung cancer. N Engl J Med 363: 1693-1703, 2010.

4. Davies JJ, Wilson IM and Lam WL: Array CGH technologies and their applications to cancer genomes. Chromosome Res 13: 237-248, 2005.

5. de Leeuw RJ, Davies JJ, Rosenwald A, et al: Comprehensive whole genome array CGH profiling of mantle cell lymphoma model genomes. Hum Mol Genet 13: 1827-1837, 2004.

6. Nakao K, Mehta KR, Fridlyand J, et al: High-resolution analysis of DNA copy number alterations in colorectal cancer by arraybased comparative genomic hybridization. Carcinogenesis 25: 1345-1357, 2004

7. Wong MP, Fung LF, Wang E, et al: Chromosomal aberrations of primary lung adenocarcinomas in nonsmokers. Cancer 97: 1263-1270, 2003.

8. Zhu H, Lam DC, Han KC, et al: High resolution analysis of genomic aberrations by metaphase and array comparative genomic hybridization identifies candidate tumour genes in lung cancer cell lines. Cancer Lett 245: 303-314, 2007.

9. Lan Q, Hsiung CA, Matsuo K, et al: Genome-wide association analysis identifies new lung cancer susceptibility loci in neversmoking women in Asia. Nat Genet 44: 1330-1335, 2012.

10. Hsiung CA, Lan Q, Hong YC, et al: The 5p15.33 locus is associated with risk of lung adenocarcinoma in never-smoking females in Asia. PLoS Genet 6: e1001051, 2010.

11. McKay JD, Hung RJ, Gaborieau V, et al: Lung cancer susceptibility locus at 5p15.33. Nat Genet 40: 1404-1406, 2008. 
12. Wang Y, Broderick P, Webb E, et al: Common 5p15.33 and 6 p21.33 variants influence lung cancer risk. Nat Genet 40: 1407-1409, 2008.

13. Dong J, Hu Z, Wu C, et al: Association analyses identify multiple new lung cancer susceptibility loci and their interactions with smoking in the Chinese population. Nat Genet 44: 895-899, 2012.

14. Fan B, Dachrut S, Coral H, et al: Integration of DNA copy number alterations and transcriptional expression analysis in human gastric cancer. PLoS One 7: e29824, 2012.

15. Holzmann K, Kohlhammer H, Schwaenen C, et al: Genomic DNA-chip hybridization reveals a higher incidence of genomic amplifications in pancreatic cancer than conventional comparative genomic hybridization and leads to the identification of novel candidate genes. Cancer Res 64: 4428-4433, 2004

16. Tonon G, Wong KK, Maulik G, et al: High-resolution genomic profiles of human lung cancer. Proc Natl Acad Sci USA 102: 9625-9630, 2005

17. Kim TM, Yim SH, Lee JS, et al: Genome-wide screening of genomic alterations and their clinicopathologic implications in non-small cell lung cancers. Clin Cancer Res 11: 8235-8242, 2005.

18. Shibata T, Uryu S, Kokubu A, et al: Genetic classification of lung adenocarcinoma based on array-based comparative genomic hybridization analysis: its association with clinicopathologic features. Clin Cancer Res 11: 6177-6185, 2005.

19. Rácz A, Brass N, Höfer M, Sybrecht GW, Remberger K and Meese EU: Gene amplification at chromosome 1pter-p33 including the genes PAX7 and ENO1 in squamous cell lung carcinoma. Int J Oncol 17: 67-73, 2000.

20. Xu X, Padilla MT, Li B, et al: MUC1 in macrophage: contributions to cigarette smoke-induced lung cancer. Cancer Res 74: 460-467, 2014
21. Yan C, Ding X, Wu L, Yu M, Qu P and Du H: Stat3 downstream gene product chitinase 3-like 1 is a potential biomarker of inflammation-induced lung cancer in multiple mouse lung tumor models and humans. PLoS One 8: e61984, 2013.

22. Aras G, Kanmaz D, Urer N, Purisa S, Kadakal F, Yentürk E and Tuncay E: Immunohistochemical expression of telomerase in patients with non-small cell lung cancer: prediction of metastasis and prognostic significance. Anticancer Res 33: 2643-2650, 2013.

23. Lantuéjoul S, Salon C, Soria JC and Brambilla E: Telomerase expression in lung preneoplasia and neoplasia. Int J Cancer 120: 1835-1841, 2007.

24. Pizzi M, Fassan M, Balistreri M, Galligioni A, Rea F and Rugge M: Anterior gradient 2 overexpression in lung adenocarcinoma. Appl Immunohistochem Mol Morphol 20: 31-36, 2012.

25. Larue L and Delmas V: The WNT/Beta-catenin pathway in melanoma. Front Biosci 11: 733-742, 2006.

26. Zhou XL, Qin XR, Zhang XD and Ye LH: Downregulation of Dickkopf-1 is responsible for high proliferation of breast cancer cells via losing control of Wnt/ $\beta$-catenin signaling. Acta Pharmacol Sin 31: 202-210, 2010.

27. Gautschi O, Ratschiller D, Gugger M, Betticher DC and Heighway J: Cyclin D1 in non-small cell lung cancer: a key driver of malignant transformation. Lung Cancer 55: 1-14, 2007.

28. Gee JM, Eloranta JJ, Ibbitt JC, et al: Overexpression of TFAP2C in invasive breast cancer correlates with a poorer response to anti-hormone therapy and reduced patient survival. J Pathol 217: 32-41, 2009.

29. Lee MH and Surh YJ: eFF1A2 as a putative oncogene. Ann NY Acad Sci 1171: 87-93, 2009. 